\title{
Flexible and Self-adaptive Sense-and-Compress for sub-microWatt always-on sensory recording
}

\author{
Jaro De Roose*, Haoming Xin ${ }^{\dagger}$, Martin Andraud*, Pieter J. A. Harpe ${ }^{\dagger}$, Marian Verhelst* \\ ${ }^{*}$ KU Leuven, Dept. of Electrical Engineering (ESAT), MICAS group, Belgium \\ \{jaro.deroose,martin.andraud,marian.verhelst\} @esat.kuleuven.be \\ ${ }^{\dagger}$ Eindhoven University of Technology, Depart. of Electrical Engineering, MSM Group, The Netherlands \\ \{h.xin,p.j.a.harpe\}@tue.nl
}

\begin{abstract}
Miniaturized sensory systems for IoT applications experience a severe power burden from their wireless link and/or embedded storage system. Compressive sensing techniques target data compression before storage and transmission to save power, while minimizing information loss. This work proposes a selfadaptive sense-and-compress system, which consumes only 45884nW while continuously recording and compressing signals with a bandwidth up to $5 \mathrm{kHz}$. The flexible system uses a combination of off-line Evolutionary Algorithms, and on-line self-adaptivity to constantly adapt to the incoming sensory data statistics, and the current application quality requirements. The $0.27 \mathrm{~mm}^{2}$ sense-and-compress interface is integrated in a $65 \mathrm{~nm}$ CMOS technology, together with an on-board temperature sensor, or can interface with any external sensor. The scalable, selfadaptive system is moreover heavily optimized for low-power and low-leakage, resulting in a tiny, efficient, yet flexible interface allowing always-on sensory monitoring, while consuming $\mathbf{2 . 5 X}$ less power compared to the current State-of-the-Art.
\end{abstract}

\section{INTRODUCTION}

The push for miniaturization of Internet of Things (IoT) systems demands smaller batteries and hence more power efficient sensor systems. An IoT sensor system, depicted in Fig. 1, locally captures and digitizes sensory data from various sensors. Data transfer to the base computer can be implemented through temporary storage in non-volatile memories, or by RF transmission to the base computer. This data transfer however strongly dominates the power consumption of a typical IoT sensor system. Therefore, State-of-the-Art techniques (SotA) reduce the data rate by compressing sensory data on chip before storing/sending it [1]-[3]. Such compression saves power, yet distorts the measured signal and introduces loss of sensory information (Infoloss), which can be expressed in terms of Percentage Root-mean-square Distortion (PRD) = RMS (error signal) / RMS(original signal), where the error signal is the difference between the uncompressed original signal and the
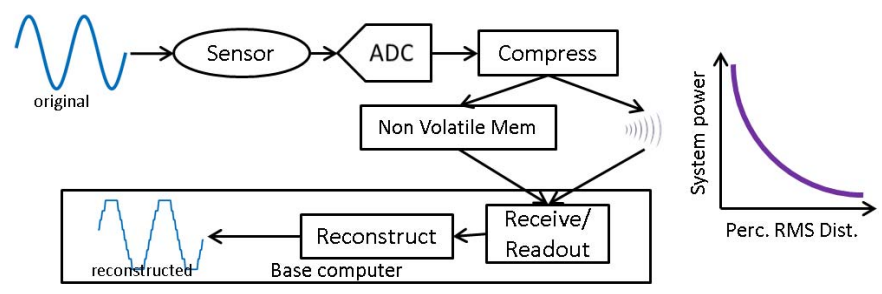

Fig. 1. IoT sensor data gathering system reconstructed signal.

SotA techniques all try to maximize compression while keeping PRD within application requirements. Compressed sensing (CS) [1] transforms and subsamples data, exploiting the signal's sparsity characteristics. Yet, the simple compressive interface is unaware of the incoming sensory signals and unable to adapt to varying signal sparsity or statistics. Online adaptivity is achieved in [2] through an asynchronous ADC, self-adapting its sampling rate to the slope of the incoming signal. Yet, again, adaptivity is limited, only taking the steepness of the data into account. Improved online adaptation for improved compression efficiency is demonstrated in [3], through temporal decimation and wavelet shrinkage. Yet, the system is limited to ECG signals, making use of their specific properties.

Research [4] indicates that no single compression technique performs optimally across all data types, limiting the versatility and real world efficiency of dedicated and rigid SotA solutions. Moreover, most SotA solutions try to blindly maximize compression rate under PRD constraints, while instead total end-to-end system power is what matters. This however requires to closely co-optimize power spent in the analog front end, the compression block itself and in data transfer.

This work overcomes these shortcomings through:

1) a flexible sense-and-compress system optimized for flexibility and low-leakage (Section II).

2) Online reconfiguration of the system based on online sensory data statistics (section III)

3) Automated off-line pre-optimization, using an Evolutionary Algorithm (EA) and training data (Section IV).

These three innovations, together with an extensive full-system power model (analog, digital and transfer) result in a senseand-compression system usable across a wide range of sensing applications. The approach allows to optimize total system power consumption of the complete IoT node, in function of application and data dependent PRD requirements. This will be demonstrated with measurements in Section V.

\section{FLEXIBLE SENSE-AND-COMPRESS SYSTEM}

The proposed flexible sense-and-compress system is depicted in Fig. 2. It integrates all components from the sensor to the compressed output, which can subsequently be stored in nonvolatile memory or be sent out via a wireless link. The senseand-compress system can be used with several sensor types. 


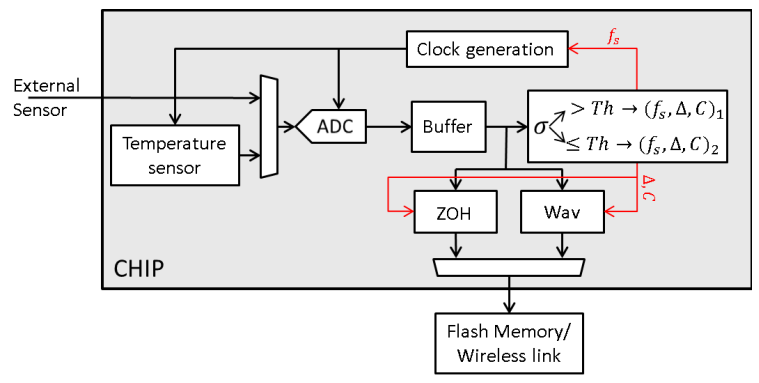

Fig. 2. Top-level architecture: Sensor interface with external sensor and on-chip temperature sensor, SAR ADC, sample buffer, clock generation, and powergated $\mathrm{ZOH}$ and Wavelet compression

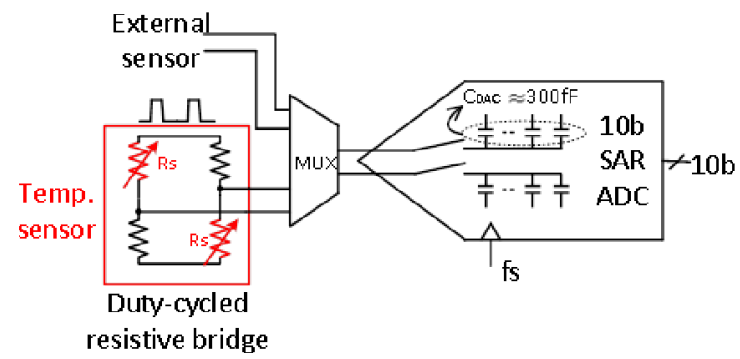

Fig. 3. The sensor interface. External sensor signal and duty-cycled resistive temperature sensor digitized by a $10 \mathrm{~b}$ SAR ADC.

As a proof-of-concept, it has been designed to operate on data coming either from a built-in resistive temperature sensor, or from an external sensor interface. A 10b asynchronous SAR ADC (Fig. 3) converts this sensory signal into the digital domain, and the converted samples are subsequently stored in a buffer. When the buffer is full, the signal is compressed in one of two available parametrized compression blocks. Finally, a memory interface transfers the compressed blocks of data in a burst to an external flash memory or wireless interface. All components are optimized for low power, low leakage operation and high flexibility.

a) Sensor and ADC: The duty-cycled resistive bridge and dynamic ADC [5] consume only dynamic power, which scales proportionally to the actual sampling frequency $\left(\mathrm{f}_{s}\right)$. The small ADC capacitance minimizes the dynamic energy while HVT transistors with increased length are used in the ADC logic to reduce leakage. The adaptive ADC sampling frequency $\mathrm{f}_{s}$ is generated on-chip, controlled through a programmable counter.

b) Compression subsystem: The compression type can be varied online, through the implementation of both 1.) ZeroOrder-Hold $(\mathrm{ZOH})$ compression, which discards all samples which differ less than $\Delta$ from the previously stored sample; and 2.) Wavelet compression, which discards all wavelet coefficients with an amplitude below $\Delta$ [6]. In both subblocks $\Delta$ hence determines the harshness of compression, impacting PRD, as exploited in Section IV.

c) Buffer for leakage optimization: The slow nature of IoT sensory data makes the digital subsystem dominated by leakage rather than dynamic power. Therefore, the dominant blocks in terms of leakage (the compression blocks and the non-volatile memory) are powergated while the incoming

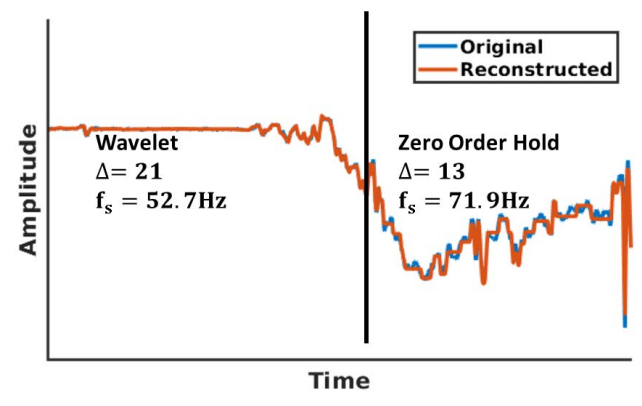

Fig. 4. Illustration of online changing between compression settings

sensory data is captured in a small 256-entry buffer, reducing total chip leakage below 50nW. The samples in the buffer are compressed and stored in the memory in a burst at high frequency, so that the blocks can quickly go back to sleep mode.

\section{RESOURCE-EFFICIENT SELF-ADAPTATION}

As discussed in Section II, the implemented system is made run-time configurable along three tuning parameters: the compression algorithm $(\mathrm{C})$, compression harshness $(\Delta)$ and sample frequency $\left(\mathrm{f}_{s}\right)$. These parameters can be updated each time the sample buffer is full, enabling a dynamic tradeoff between PRD and total system power. The desired signaldependent operation is achieved through multiple sets of tuning parameters $\mathrm{f}_{s}, \mathrm{C}$ and $\Delta$ present in the chip, between which the chip can switch at runtime. To this extent, after each full buffer, statistical parameters of the samples inside the buffer are calculated, such as mean, variance, or higher order statistics. Based on these statistical parameters, a specific set of tuning parameters is selected and applied to the circuit. Using the statistical parameters, it is possible to detect different online scenarios, and apply diversified tuning parameters. This allows, for example, to treat high and low variance signals differently. The advantages are twofold. Firstly, it is possible to optimize the tuning parameters orthogonally for data with different statistical properties, increasing the overall efficiency. Secondly, it is possible to tolerate higher PRD on some uninteresting parts of the data, reducing the power consumption on this data. This chip, as a proof-of-concept, implemented this approach assuming two tuning parameter sets, while using variance as the decisive runtime statistical property of the input data. Specifically, when the buffer is full, the variance of the samples in the buffer is calculated and compared to a threshold $T H$. Two sets of parameters $f_{s}, C$ and $\Delta$ are present, one for $>T H$ and one for $\leq T H$. Fig. 4 shows an example: when the variance becomes higher, the compression switches from Wavelet compression to $\mathrm{ZOH}$ compression with different tuning parameters. This e.g. allows to accurately store highly volatile movement data, while not wasting power on quiet signals. Note that the presented concept is not limited to 2 sets, nor to variance-based data statistics. This powerful approach allows to push down power consumption at runtime, in function of the statistics and interest in the signal present. 

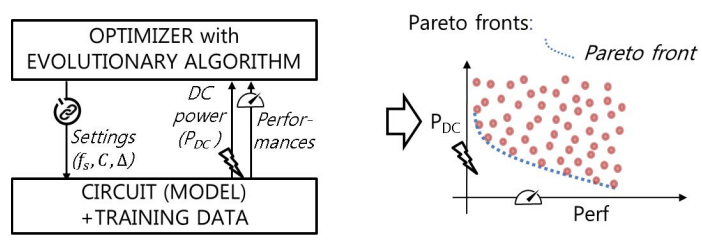

Fig. 5. Evolutionary Algorithm mechanics

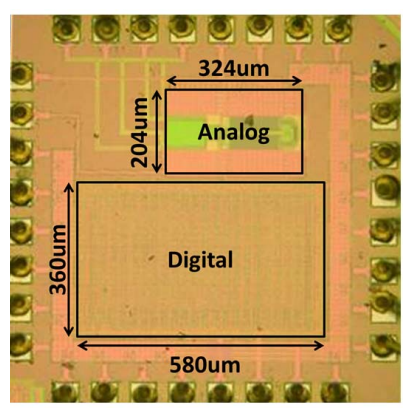

\begin{tabular}{|l|r|}
\hline System & $\begin{array}{r}\text { Temperature sensor, sensor } \\
\text { interface, SAR ADC, } \\
\text { compression blocks, } \\
\text { intelligence }\end{array}$ \\
\hline Technology & 65nm Low Power CMOS \\
\hline Area & $0.2749 \mathrm{~mm}^{2}$ \\
\hline Gate count & 15209 \\
\hline $\begin{array}{l}\text { Sample } \\
\text { frequency range }\end{array}$ & $0 \mathrm{~Hz}$ to $10 \mathrm{kHz}$ \\
\hline $\begin{array}{l}\text { Compression } \\
\text { algorithms }\end{array}$ & Zero-Order-Hold (ZOH) \\
\hline Delta (A) range & Wavelet (Wav) \\
\hline ADC Resolution & 0 to 1023 \\
\hline Vdd & 10 bit \\
\hline Power & $0.66 \mathrm{~V}$ (ZOH), $0.76 \mathrm{~V}$ (Wav) \\
\hline \multicolumn{2}{|c|}{$45 \rightarrow 884 \mathrm{nW}$} \\
\hline
\end{tabular}

Fig. 6. Chip Photograph and system properties table

\section{OFFLINE PRE-OPTIMIZATION USING EA}

Each application has a different optimal set of tuning parameters $T H, f_{s,<T H}, C_{<T H}, \Delta_{<T H}$ and $f_{s,>T H}, C_{>T H}$, $\Delta_{>T H}$. These optimal parameters should be determined offline to save online resources. They should depend strongly on the expected data statistics, the applications PRD requirements and the systems total power consumption. An automated approach is used to make the presented concept quickly adapt to different applications with no design effort. To this end, the work uses an Evolutionary Algorithm (EA) and a power-performance model of the flexible hardware (including power for the storage memory or wireless interface) and previously gathered representative (not identical) data (=training data), representing the sensing scenario. The EA creates different generations of tuning parameters $\left(T H, f_{s,<T H}, C_{<T H}, \Delta_{<T H}\right.$ and $f_{s,>T H}$, $C_{>T H}, \Delta_{>T H}$ ), evaluating PRD and power impact on the training data for each generation as a fitness function to create the next generation. This allows to converge to the subsets forming the Pareto-front with the best power-PRD trade-off, as shown in Fig. 5. When changing the model of hardware or training data and rerunning the EA, a new Pareto front is automatically created, which adapts to these changes.

\section{Measurement Results}

The sensor interface, ADC and the adaptive sense-andcompress block have been jointly integrated in a $65 \mathrm{~nm}$ LP CMOS technology. It has a total active area of $0.066 \mathrm{~mm}^{2}$ and $0.209 \mathrm{~mm}^{2}$ for analog and digital respectively (Fig. 6).

\section{A. System flexibility and low-power operation}

a) Sensor performance: The built-in temperature sensor consumes down to $4.88 \mathrm{pJ}$ per sample at a $0.65 \mathrm{~V}$ supply for the resistive bridge and SAR ADC, and a $1 \mathrm{~V}$ supply for the clock drivers. It achieves a rms resolution of $0.61^{\circ} \mathrm{C}$, and a minimum/maximum inaccuracy of $-1.1 / 1.5^{\circ} \mathrm{C}$ from $0-100^{\circ} \mathrm{C}$ over 12 IC samples.

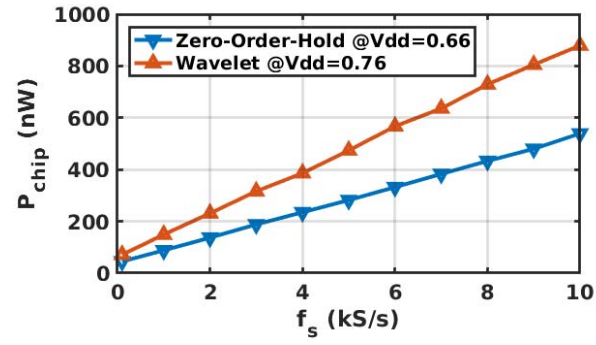

Fig. 7. Chip power consumption. Zero Order Hold stand-alone consumes less power due to lower minimal $\mathrm{V}_{D D}$

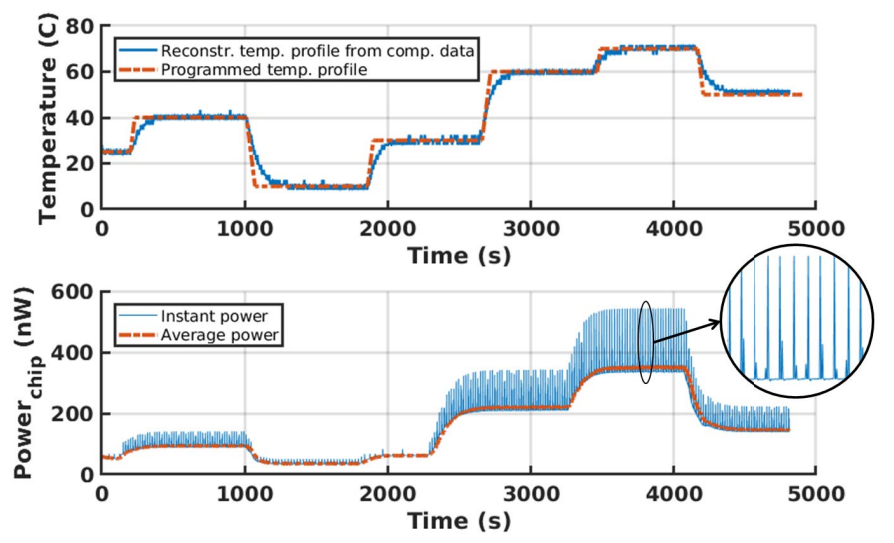

Fig. 8. Temperature measurement (top), Power consumption (bottom)

b) System power-scalability and flexibility: Fig. 7 shows the power consumption of the full system in function of $f_{s}$ and $C$. The $\mathrm{ZOH}$ algorithm is intrinsically less complex than Wavelet compression, allowing reduction of $V_{D D}$ and power. The chip can sample from 0 to $10 \mathrm{kHz}$ with 10 bit accuracy, which suffices for most IoT sensory data.

c) Ultra-low power full-system operation: The complete system with internal temperature sensor is placed in a temperature room with varying temperature as shown in Fig. 8 to assess the power consumption of the complete system in a practical application. The chip senses, digitizes and compresses the temperature sensor data stream, while consuming only $151 \mathrm{nW}$. When combined with a low-power flash memory [7] the complete application would run with less than $200 \mathrm{nW}$. In this application, power consumption is completely dominated by temperature dependent leakage power, as seen in the bottom of Fig. 8. The power consumption shows spikes when the buffer is full and the calculation core is activated.

\section{B. Adaptation and optimization}

To prove the systems self-adaptivity and automation capabilities, we apply our system on Human-Activity-Recognition (HAR) accelerometer data [8], constructing the dataset with 2 hours of human activity a day. The EA loop offline optimizes the settings for low and high variance data on training data, finding a Pareto-front of the best trade-off settings between complete system power consumption and PRD, as highlighted in Fig. 9. Each dot in the plot is a Pareto-optimum TH, $\mathrm{f}_{s,<T H}$, $\mathrm{C}_{<T H}, \Delta_{<T H}$ and $\mathrm{f}_{s,>T H}, \mathrm{C}_{>T H}, \Delta_{>T H}$ combination, from which one can be selected, depending on the application 

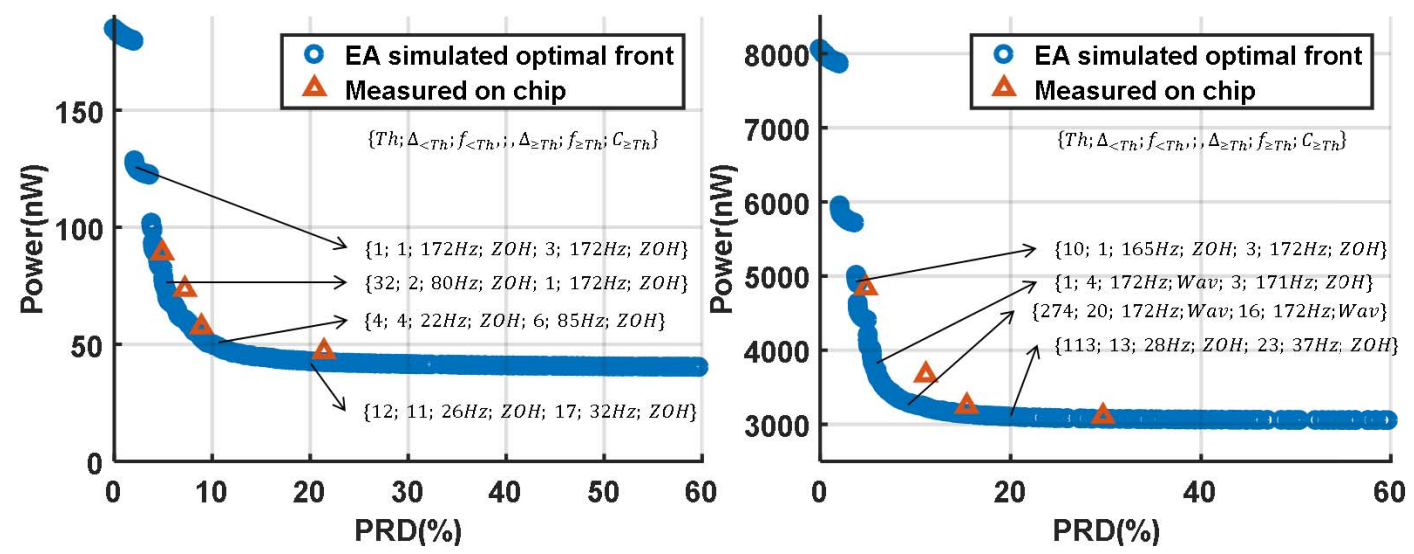

Fig. 9. Measurement results with low power flash (left) and commercial flash (right) memory model. Changing the memory model changes the optimal settings

TABLE I

PERFormance OVERVIEW STATE-OF-THE-ART. * ARE DOMINATED By LEAKAGE, MAKING THEM NON-SCALABLE TO LOWER SAMPLING FREQUENCIES

\begin{tabular}{|c|c|c|c|c|c|}
\hline & 2017 IEEE [3] & 2012 JSSC [1] & 2011 IEEE [2] & This work (high perf) & This work (low power) \\
\hline System & Dig. backend & ADC, Dig. backend & Flexible ADC & \multicolumn{2}{|c|}{ Sensor interface, ADC, Dig. Backend } \\
\hline Technique & Wavelet Shrinkage + Temp. decimation & Compressed sensing & Asynchronous ADC & \multicolumn{2}{|c|}{ Adaptive compression } \\
\hline Run time adaptation & Yes & No & Slope adaptation & \multicolumn{2}{|c|}{ Yes } \\
\hline Adapt to application & No (ECG only) & No & No & \multicolumn{2}{|c|}{ Yes } \\
\hline Technology & $180 \mathrm{~nm} \mathrm{CMOS}$ & $90 \mathrm{~nm}$ CMOS & $180 \mathrm{~nm} \mathrm{CMOS}$ & \multicolumn{2}{|c|}{$65 \mathrm{~nm}$ CMOS } \\
\hline Area & $0.86 \mathrm{~mm}^{2}$ & $0.104 \mathrm{~mm}^{2} \mathrm{dig}+\mathrm{ana}$ & $0.96 \mathrm{~mm}^{2}$ & \multicolumn{2}{|c|}{$0.066 \mathrm{~mm}^{2}$ ana, $0.209 \mathrm{~mm}^{2} \mathrm{dig}$} \\
\hline Resolution & 12 bit & 8 bit & Up to 8 bit & \multicolumn{2}{|c|}{ 10bit ZOH, 9bit Wav } \\
\hline Vdd & $0.45 \mathrm{~V}$ & $0.6 \mathrm{~V}$ & $0.7 \mathrm{~V}$ & \multicolumn{2}{|c|}{$0.66 \mathrm{~V}(\mathrm{ZOH}), 0.76 \mathrm{~V}(\mathrm{Wav})$} \\
\hline Sample speed & 60 to $1000 \mathrm{~S} / \mathrm{s}$ & Up to $20 \mathrm{kS} / \mathrm{s}$ & Up to $50 \mathrm{kS} / \mathrm{s}$ & up to $10 \mathrm{kS} / \mathrm{s}$ & Up to $1 \mathrm{kS} / \mathrm{s}$ \\
\hline Power & 147 to $375 \mathrm{nW}$ & $1.9 \mathrm{uW}$ dig* & $25 \mathrm{uW}$ ana* & 544nW(ZOH), 884nW(Wav) & 91nW(ZOH), 146nW (Wav) \\
\hline
\end{tabular}

constraints. Note that the optimal settings are different for a system interfacing with a low-power flash memory [7] (left) resp. a commercial LE25S161 flash memory (right). As such, the automated approach allows to quickly and optimally customize to various system configurations, such as the type of storage or wireless link. E.g., as seen from Fig. 9, wavelet compression only becomes interesting when the flash memory dominates the complete system power, making a more complex and expensive compression algorithm more attractive. Next, a Pareto combination is loaded into the chip, which is fed with (different) test data (different from the training data), from which resulting measured performance for several Pareto settings is shown in Fig. 9.

\section{CONCLUSION}

The presented flexible, self-adaptive sense-and-compress system is compared to the state-of-the-art in Table I. This work is the only complete system integrating the full sensor frontend and digital compression back-end together, while being able to continuously self-adapt towards the most efficient data compression. Low power and low leakage design allows to record data with $<150 \mathrm{nW}$ power when sampling up to $1 \mathrm{kS} / \mathrm{s}$ and $<1 \mu \mathrm{W}$ up to $10 \mathrm{kS} / \mathrm{s}$.

\section{ACKNOWLEDGEMENTS}

This project has received funding from the EU H2020 research and innovation programme, grant agreement No665347.

\section{REFERENCES}

[1] F. Chen, A. P. Chandrakasan, and V. M. Stojanovic, "Design and analysis of a hardware-efficient compressed sensing architecture for data compression in wireless sensors," IEEE Journal of Solid-State Circuits, vol. 47, no. 3, pp. 744-756, March 2012.

[2] M. Trakimas and S. R. Sonkusale, "An adaptive resolution asynchronous ADC architecture for data compression in energy constrained sensing applications," IEEE Transactions on Circuits and Systems I: Regular Papers, vol. 58, no. 5, pp. 921-934, May 2011.

[3] C. I. Ieong et al., "A $0.45 \mathrm{~V} 147-375 n W$ ECG compression processor with wavelet shrinkage and adaptive temporal decimation architectures," IEEE Transactions on Very Large Scale Integration (VLSI) Systems, vol. 25, no. 4, pp. 1307-1319, April 2017.

[4] M. Andraud et al., "Exploring the unknown through successive generations of low power and low resource versatile agents," in Design, Automation Test in Europe Conference Exhibition (DATE), 2017, March 2017, pp. 290-293.

[5] H. Xin, M. Andraud, P. Baltus, E. Cantatore, and P. Harpe, "A 174pW$488.3 \mathrm{nW} 1 \mathrm{~S} / \mathrm{s}-100 \mathrm{kS} / \mathrm{s}$ all-dynamic resistive temperature sensor with speed/resolution/resistance adaptability," in IEEE solid-state circuit letters, April 2018, in press.

[6] A. Hallawa, J. De Roose, M. Andraud, M. Verhelst, and G. Ascheid, "Instinct-driven dynamic hardware reconfiguration: Evolutionary algorithm optimized compression for autonomous sensory agents," Genetic and Evolutionary Computation Conference, July 2017.

[7] Q. Dong et al., "A $1 \mathrm{Mb}$ embedded NOR flash memory with $39 \mu \mathrm{W}$ program power for mm-scale high-temperature sensor nodes," in 2017 IEEE International Solid-State Circuits Conference (ISSCC), Feb 2017, pp. 198-199.

[8] A. Stisen et al., "Smart devices are different: Assessing and mitigatingmobile sensing heterogeneities for activity recognition," in Proceedings of the 13th ACM Conference on Embedded Networked Sensor Systems, ser. SenSys '15. New York, NY, USA: ACM, 2015, pp. 127-140. 